\title{
Time-Lapse Dynamics of the Mouse Oocyte Chromatin Organisation during Meiotic Resumption
}

\author{
Martina Belli, ${ }^{1}$ Giulia Vigone, ${ }^{1}$ Valeria Merico, ${ }^{1}$ Carlo Alberto Redi, ${ }^{1,2}$ \\ Silvia Garagna, ${ }^{1,3}$ and Maurizio Zuccotti ${ }^{4}$ \\ ${ }^{1}$ Laboratorio di Biologia dello Sviluppo, Dipartimento di Biologia e Biotecnologie "Lazzaro Spallanzani", \\ Università degli Studi di Pavia, Via Ferrata 9, 27100 Pavia, Italy \\ ${ }^{2}$ Centro Ricerche di Medicina Rigenerativa, Fondazione IRCCS Policlinico San Matteo, 27100 Pavia, Italy \\ ${ }^{3}$ Centro di Ingegneria Tissutale, Università degli Studi di Pavia, 27100 Pavia, Italy \\ ${ }^{4}$ Sezione di Anatomia, Istologia ed Embriologia, Dipartimento di Scienze Biomediche, \\ Biotecnologiche e Traslazionali (S.Bi.Bi.T.), Università degli Studi di Parma, Via Volturno 39, 43125 Parma, Italy
}

Correspondence should be addressed to Silvia Garagna; silvia.garagna@unipv.it and Maurizio Zuccotti; maurizio.zuccotti@unipr.it

Received 10 January 2014; Accepted 25 February 2014; Published 30 March 2014

Academic Editor: John Huntriss

Copyright (C) 2014 Martina Belli et al. This is an open access article distributed under the Creative Commons Attribution License, which permits unrestricted use, distribution, and reproduction in any medium, provided the original work is properly cited.

In the mammalian oocyte, distinct patterns of centromeres and pericentromeric heterochromatin localisation correlate with the gamete's developmental competence. Mouse antral oocytes display two main types of chromatin organisation: SN oocytes, with a ring of Hoechst-positive chromatin surrounding the nucleolus, and NSN oocytes lacking this ring. When matured to MII and fertilised, only SN oocytes develop beyond the 2-cell, and reach full term. To give detailed information on the dynamics of the SN or NSN chromatin during meiosis resumption, we performed a $9 \mathrm{hr}$ time-lapse observation. The main significant differences recorded are: (1) reduction of the nuclear area only in SN oocytes; (2) 17 min delay of GVBD in NSN oocytes; (3) chromatin condensation, after GVBD, in SN oocytes; (4) formation of 4-5 CHCs in SN oocytes; (5) increase of the perivitelline space, 57 min later in NSN oocytes; (6) formation of a rosette-like disposition of $\mathrm{CHCs}, \sim 84 \mathrm{~min}$ later in SN oocytes; (7) appearance of the MI plate $\sim 40 \mathrm{~min}$ later in NSN oocytes. Overall, we described a pathway of transition from the GV to the MII stage that is punctuated of discrete recordable events showing their specificity and occurring with different time kinetics in the two types of oocytes.

\section{Introduction}

In the nucleus of eukaryotic cells, chromosomes occupy distinct territories whose position may change during the cell cycle or cell differentiation [1-4]. Entire chromosomes, subchromosomal regions, and genes change their nuclear localisation during differentiation to acquire a cell-type-specific spatial organisation, contributing, as part of the epigenome, to the regulation of the cell functions [5-8]. Centromeres and pericentromeric constitutive heterochromatin $(\mathrm{CHC})$ of mammalian cells tend to gather to form chromocenters. The degree of centromere clustering varies depending on the cell type, cell-cycle phase, or stage of differentiation [9-14]. Lineage-specific centromere associations into chromocenters have been reported during somatic $[9,15,16]$, male [17], and female $[18,19]$ germ cell differentiation. Within the nucleus, the nucleolus is a major attractive compartment for heterochromatic regions such as inactive $\mathrm{X}$-chromosome, regions enriched in repressed genes, and pericentromeric repeated sequences [20]. Altogether, these studies indicate that a specific nuclear localisation of these chromatin traits is required for a correct genome functioning at different cellcycle phases and different stages of cell differentiation [21].

To this regard, the mammalian oocyte is a particularly intriguing cell model study as distinct patterns of centromeres and pericentromeric $\mathrm{CHC}$ localisation correlate with the gamete meiotic and developmental competence. When stained with Hoechst 33342 (Ho), a supravital fluorochrome that preferentially binds to the AT-rich regions of the genome, fully grown germinal vesicle (GV) mouse 
antral oocytes display two main different types of chromatin organisation: Surrounded Nucleolus (SN) oocytes, with a ring of Ho-positive chromatin surrounding the nucleolus and Nonsurrounded Nucleolus (NSN) oocytes, lacking the ring and with a more dispersed chromatin [22-24]. A bold of experimental evidence has shown that, following their isolation from the ovarian surface, SN and NSN antral oocytes display a different meiotic competence, with $82 \%$ or $45 \%$ of $\mathrm{SN}$ and NSN oocytes, respectively, reach the metaphase II (MII) stage [25]. Then, after fertilisation, only SN oocytes may develop to term, whereas NSN oocytes arrest at the 2-cell stage [25-28].

The two different nuclear phenotypes underlie specific transcriptional and translational programmes central to the acquisition of a correct developmental competence or developmental failure [29-32]. Their distinct chromatin organisations, which are found in most of the mammals studied (for a review see [33]), reflect the pattern of localisation and arrangements of the nucleolar organising region- (NOR-) bearing chromosomes and of the nuclear and perinucleolar localisation of centromeres and their associated pericentromeric CHC occurring during folliculogenesis $[18,19]$.

The crux of the present study is to contribute detailed information on the morphological transformations occurring to the nuclear organisation of the SN or NSN chromatin during meiosis resumption. We conducted a live observation of GV oocytes while progressing in vitro towards the MII stage, with the aim of giving an accurate description of the dynamics of this transition. To this end, we isolated fully grown antral oocytes from the ovaries of females primed with PMSG and, after staining with Ho, we performed a comprehensive $9 \mathrm{hr}$ time-lapse imaging of both SN and NSN oocytes. The results describe a pathway of transition from the GV to the MII stage that is punctuated of discrete recordable events showing their specificity and occurring with different time kinetics in the two types of oocytes.

\section{Materials and Methods}

2.1. Animals. Four- to six-week-old CD1 female mice were used for this study (Charles River, Como, Italy). Mice were maintained by the Department of Animal Facility according to the Guide for Care and Use of Laboratory Animals, under conditions of $21^{\circ} \mathrm{C}$ temperature and a dark/light cycle of 12/12 hours. This research has been performed after the approval of the Animal Ethics Committee of the University of Pavia and carried out in strict accordance with the protocol approved by the European (n. 86/609/CEE) and Italian (n. 116/92, 8/94) legislation.

2.2. Hormonal Treatment. Forty-eight hours before sacrifice and oocytes isolation, female mice were intraperitoneally injected with 3.75 IU Folligon (PMSG, Intervet Italia, Segrate, Italy).

2.3. Isolation of Fully Grown Antral Oocytes. Fully-grown antral oocytes were collected in M2 medium by puncturing the ovarian surface with a sterile insulin needle. Then, they were freed from surrounding cumulus cells by gently pipetting in and out with a mouth-controlled hand-pulled glass Pasteur micropipette.

2.4. Classification and Maturation of Antral Oocytes to the MII Stage. Immediately, after their isolation form the ovary, cumulus-free antral oocytes were transferred into $20 \mu \mathrm{L}$ droplets of Ho fluorochrome $(0.05 \mu \mathrm{g} / \mathrm{mL}$; Sigma-Aldrich, Milano, Italy, cat. N. B2261) in M2 medium for $12 \mathrm{~min}$ at room temperature. Details on the method of classification of SN or NSN oocytes are given in [24]. Three-four oocytes were transferred in each of four $2 \mu \mathrm{L} \alpha$-MEM drops (Life Technologies, Monza, Italy, cat. N. M4526) placed at the centre of a $3.5 \mathrm{~cm}$ glass-bottom Petri dish (WillCo Wells B.V., Amsterdam, The Netherlands, cat. N. GWSt-3522) and covered with mineral oil (Sigma-Aldrich, cat. N. M8410). $\alpha$-MEM was supplemented with $5 \%$ heat-inactivated foetal bovine serum (Life Technologies, cat. N. 10270106), 2 mM LGlutamine (Life Technologies, cat. N. 25030), 5 mM Taurine (Sigma-Aldrich, cat. N. T0625), and $36 \mu \mathrm{g} / \mathrm{mL}$ sodium pyruvate (Sigma-Aldrich, cat. N. P4562). Oocytes were in vitro matured (IVM) for a total of $15 \mathrm{hr}$ inside a BioStation IM (Nikon, Torino, Italy) at $37^{\circ} \mathrm{C}$, under a $5 \% \mathrm{CO}_{2}$ humidified atmosphere. At the end of the culture period, first polar body (PBI) extrusion was assessed by monitoring oocytes under an Olympus SZX9 (Olympus, Milano, Italy) stereomicroscope.

2.5. Time-Lapse Analysis. Time-lapse analysis was performed over a $9 \mathrm{hr}$ total recording. Bright field and fluorescence images of oocytes were taken at $8 \mathrm{~min}$ time intervals and for time-lapse segments of a maximum of $1.5-2 \mathrm{hr}$; this segmentation was decided after preliminary experiments indicating that an exposure to UV irradiation prolonged beyond the $2 \mathrm{hr}$ could affect the oocyte maturation. To cover the whole $9 \mathrm{hr}$ recording period, five time-lapse segments were designed: from 0 to $2 \mathrm{hr}$ (12 SN and 7 NSN oocytes were recorded), from 2 to $3.5 \mathrm{hr}$ (6 SN and $5 \mathrm{NSN}$ oocytes), from 3.5 to $5 \mathrm{hr}$ (10 SN and $12 \mathrm{NSN}$ oocytes), from 5 to $7 \mathrm{hr}$ (9 SN and 6 NSN oocytes), and from 7 to $9 \mathrm{hr}(20 \mathrm{SN}$ and 7 NSN oocytes), for a total of 57 SN and 37 NSN oocytes analysed. At each 8 min time interval, a 15 sections Z-stack, $4 \mu \mathrm{m}$ spaced, was arranged. In order to produce the movies, all the time-lapse images acquired with the BioStation during each time segment analysed were subsequently imported into the Image J software and then converted into a file avi (7 frame per second). The GV (nucleus) area was measured using the CellSens Dimension software (Olympus). Since the GV shape is never that of a circle, this software allows drawing a Region Of Interest (ROI) by connecting a number of points that are chosen by the operator and then it calculates its area $\left(\right.$ pixel $\left.^{2}\right)$.

2.6. Statistics. Differences in the timing in which meiotic events occurred were evaluated with Student's $t$-test and Mann-Whitney $U$. Changes in the nuclear area were evaluated using Student's $t$-test. Data analysed with the SigmaStat 3.5 software were considered significantly different when $P<$ 0.05 . 
TABLE 1: Timing of events occurring during meiosis resumption of antral oocytes cultured in vitro.

\begin{tabular}{|c|c|c|c|}
\hline Type of event & $\begin{array}{c}\text { Mean } \pm \text { S.D. in minutes } \\
\text { SN oocytes }\end{array}$ & NSN oocytes & $P$ value $^{*}$ \\
\hline GVBD & $34.2 \pm 8.1$ & $51.4 \pm 19.0$ & $<0.05$ \\
\hline Increase of the perivitelline space & $24.0 \pm 10.6$ & $96.0 \pm 24.0$ & $<0.001$ \\
\hline Formation of $4-5 \mathrm{CHCs}$ & $92.4 \pm 9.9$ & $\mathrm{ND}^{* *}$ & - \\
\hline Formation of $8-10$ small pericentromeric regions (rosette) & $223.2 \pm 20.8$ & $139.2 \pm 20.9$ & $<0.01$ \\
\hline MI plate & $350.2 \pm 34.6$ & $390.7 \pm 20.5$ & $<0.05$ \\
\hline Anaphase I & $483.6 \pm 33.0^{* * *}$ & ND & - \\
\hline Extrusion of polar body I and formation of MII plate & $520.7 \pm 20.7^{* * *}$ & ND & - \\
\hline
\end{tabular}

${ }^{*}$ Comparison of SN versus NSN.

${ }^{* *}$ Not detected.

${ }^{* * *}$ Calculated on $55 \%$ of oocytes that reached anaphase I within the $9 \mathrm{hr}$ recording period.

\section{Results}

In this study we observed the morphological changes that occur to NSN and SN fully grown antral GV oocytes (bright field) and to the organisation of their Ho-positive chromatin (fluorescence) during the transition towards the MII stage. The details that are given hereafter are representative of the observations made for each of the time-lapse phases recorded of those oocytes that reached MII.

3.1. Time-Lapse Imaging from 0 to 120 Minutes. During the first $2 \mathrm{hr}$ of SN oocytes culture, bright-field observations showed that the germinal vesicle break down (GVBD) occurs on average $34.2 \pm 8.1 \mathrm{~min}$ since the starting of the recording (Table 1 ). GVBD is preceded by a $12.4 \%$ reduction $(P<0.01)$ of the GV area (Figure 1(a) (ad)) and culminates with the disassembly of the nuclear envelope and dismantle of the characteristic rounded shape (Figure 1(a) (e); see Additional file 1 available online at http://dx.doi.org/10.1155/2014/207357). Around the nucleus we observed a thin and dark rim which assumes a granular pattern, that is, small black dots that first gather along the perimeter and then, with the disassembly of the nuclear envelope, move towards the nucleus area centre that remains visible, throughout the $2 \mathrm{hr}$ observation, as a hollow (nuclear hollow, NH) (Figure 1(a) (e-n) and enlargements).

Almost coincidentally with the formation of the $\mathrm{NH}(24.0$ $\pm 10.6 \mathrm{~min}$ ), we observed a sudden detachment of the zona pellucida (ZP) at one side of the oocyte (Table 1; Figure 1(a) (d), (e), arrow; Additional file 1), likely due to a slight contraction. As a consequence, the size of the perivitelline space increases and is maintained as such throughout the remaining culture period. Whilst the increase of the perivitelline space was a feature of the gametes at this stage of maturation, in three out of twelve oocytes analysed we did not record this characteristic.

When observed under ultraviolet (UV) light, the typical SN chromatin configuration (Figure 1(a) (a') and enlargement) is maintained unaltered during the first $29.1 \pm 14.0 \mathrm{~min}$ (Figure 1(a) (a'-d')); then, abruptly during the following 8 min and concomitantly with the GVBD (Figure 1(a) (e') and enlargement), we recorded an increased chromatin condensation in the area where the nucleolus was positioned (Additional file 2). During the remaining culture period, with the disappearance of the ring of Ho-positive chromatin, 4-5 large CHCs become visible (92.4 $\pm 9.9 \mathrm{~min})$ (Table 1 ) (Figure 1(a) (l'-p')), with the exception of few thread-like chromatin structures visible among the CHCs (Figure 1(a) (l'), arrowhead in enlargement).

In NSN oocytes (Figure 1(b) (a') and enlargement), the features described above displayed overall more variable patterns. The GVBD occurred at $51.4 \pm 19.0$ min since the beginning of the culture period (Table 1; Figure 1(b) (j)), significantly later $(P<0.05)$ than that of SN oocytes, although in one oocyte it occurred at $16 \mathrm{~min}$ and in another occurred as late as $72 \mathrm{~min}$. The rim of small black dots coalesced towards the centre of the $\mathrm{NH}$ at later stages of maturation compared to SN oocytes (Figure 1(b) (l) and enlargement). The size of the perivitelline space increased, but much later $(96.0 \pm 24.0 \mathrm{~min}$; Table 1$)(P<0.001)$ compared to $\mathrm{SN}$ oocytes, and only in three out of seven oocytes analysed.

Under the UV light, throughout the 0 to 120 min interval, we recorded an almost static image showing the presence of 4/5 small Ho-positive CHC around the nucleolus (Figure 1(b) (a'-p'); Additional file 3). A visible change was observed at the time of GVBD when, at the disappearance of the nucleolus, the CHCs slightly moved towards the periphery (Figure 1(b) (i') and enlargement).

3.2. Time-Lapse Imaging from 128 to 216 Minutes. The size and shape of SN oocytes during this time-lapse period remained mainly unchanged and, at the bright field, we did not observe specific marker features.

Under UV light, from the 128 min onwards, we recorded a progressive partial decondensation of the CHCs (Figure 2(a) (b'-m'); see enlargement: arrow, large $\mathrm{CHC}$; arrowhead, small CHC; Additional file 4). In NSN oocytes, following the first $120 \mathrm{~min}$ of culture when chromatin organisation does not change, chromosomes appear abruptly (139.2 $\pm 20.9 \mathrm{~min}$ of culture (Table 1; Figure 2(b) (b') and enlargement), much earlier than in SN oocytes (see below) and assume a "rosettelike" organisation (Figure 2(b) (e') and enlargement) that is maintained up to the end of this recording time segment (Additional file 5). 


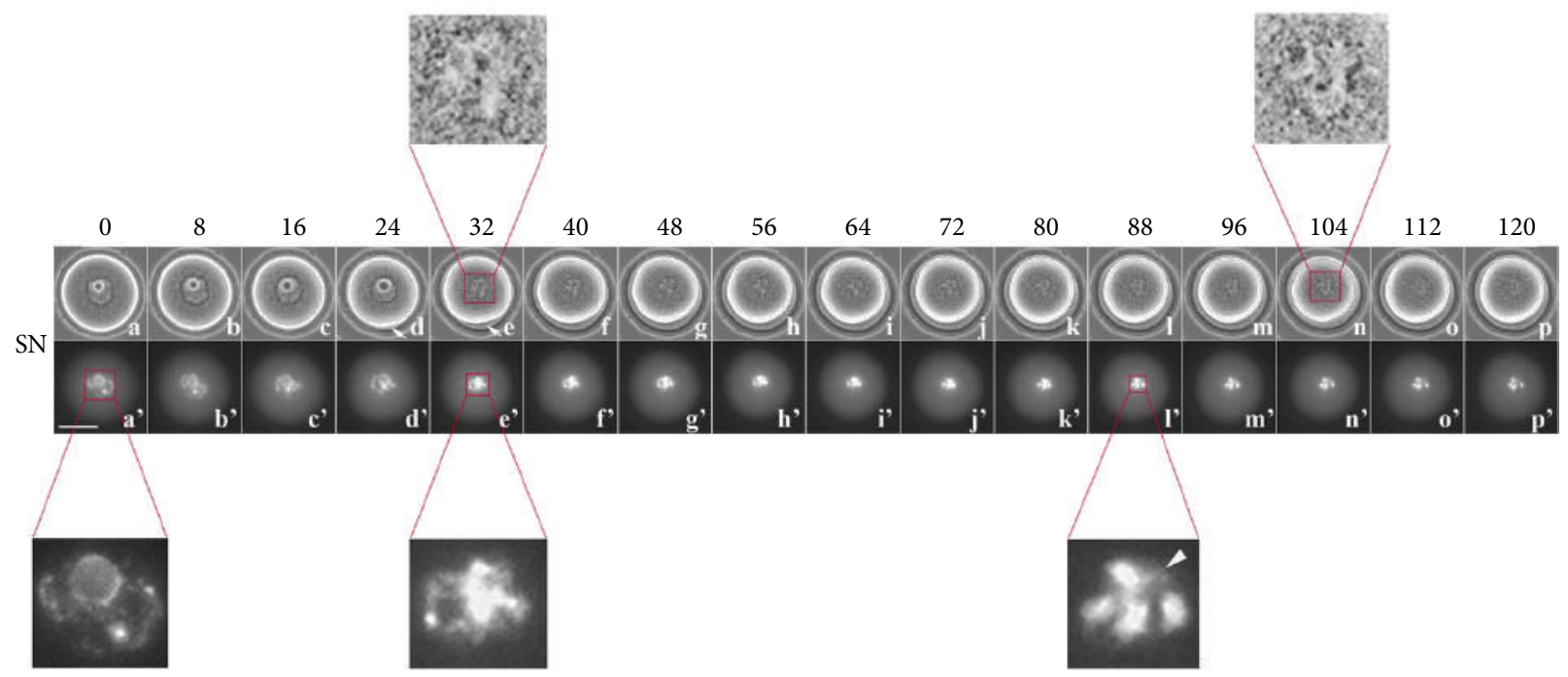

(a)

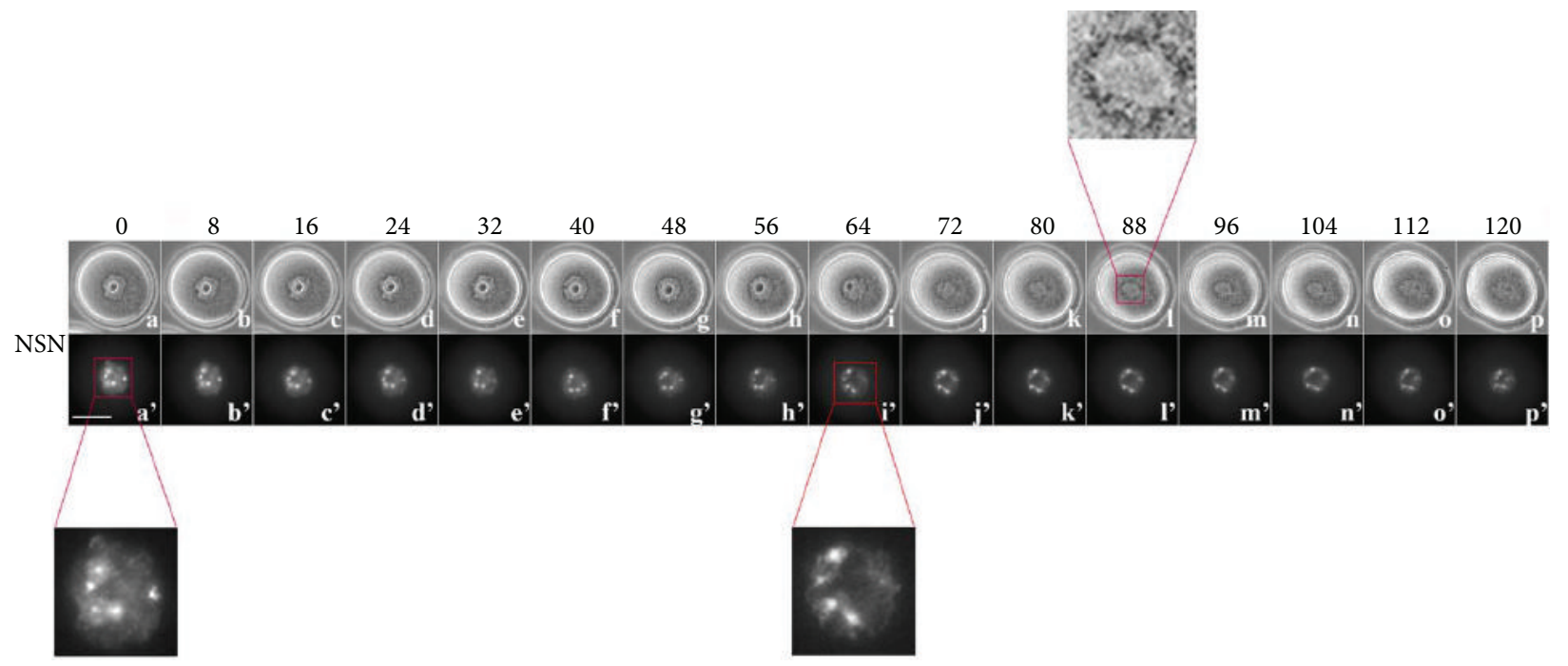

(b)

FIGURE 1: Time-lapse imaging during the first 120 minutes of oocyte culture. (a) SN oocyte, (a-p) bright field; (a'-p') UV fluorescence describing the changes to the Ho-positive chromatin within the nucleus. Arrow in (e) and (d) shows increase of perivitelline space. Arrowhead in (l') enlargement thread-like chromatin structures is visible among the pericentromeric CHCs. (b) NSN oocyte, (a-p) bright field; (a'-p') UV fluorescence. Bar: $40 \mu \mathrm{m}$.

3.3. Time-Lapse Imaging from 224 to 296 Minutes. In SN oocytes, 8-10 Ho-positive regions arrange to form a "rosettelike" distribution after $223.2 \pm 20.8$ min culture (Table 1 ; Figure 3(a) (b'-k') and enlargement), later $(P<0.01)$ than NSN oocytes. This organisation is maintained throughout this recording time segment (Additional file 6).

Chromosomes of NSN oocytes maintain the "rosettelike" disposition acquired during almost the whole period (Figure 3(b) (b'-k')), although, by the end, they begin moving towards the oocyte surface (Additional file 7).

3.4. Time-Lapse Imaging from 304 to 416 Minutes. This timelapse segment is characterised by the formation of a clear MI plate, which appears earlier $(P<0.05)$ in SN $(350.2 \pm$
34.6 min, Figure 4(a) (d') and enlargement) than NSN (390.7 $\pm 20.5 \mathrm{~min}$, Figure 4(b) (i') and enlargement) oocytes. The MI plate changes localisation in both SN (Additional file 8) and NSN (Additional file 9) oocytes (Figure 4(a) (d'-p') and Figure 4(b) (i'-p')).

3.5. Time-Lapse Imaging from 424 to 536 Minutes. Anaphase I (Table 1; Figure 5(a) (b'), Figure 5(b) (h') and enlargements), with two separated chromosome sets and PBI extrusion (SN: Table 1; Figure 5(a) (h)) (Additional files 10 and 11), is the main feature of this time-lapse segment. Although all the SN or NSN oocytes analysed reached the MII phase, the latter was attained before the end of the $9 \mathrm{hr}$ recording period (480 $\mathrm{min}$ ) by $55.0 \% \mathrm{SN}$ or $14.3 \%$ NSN oocytes, respectively. 


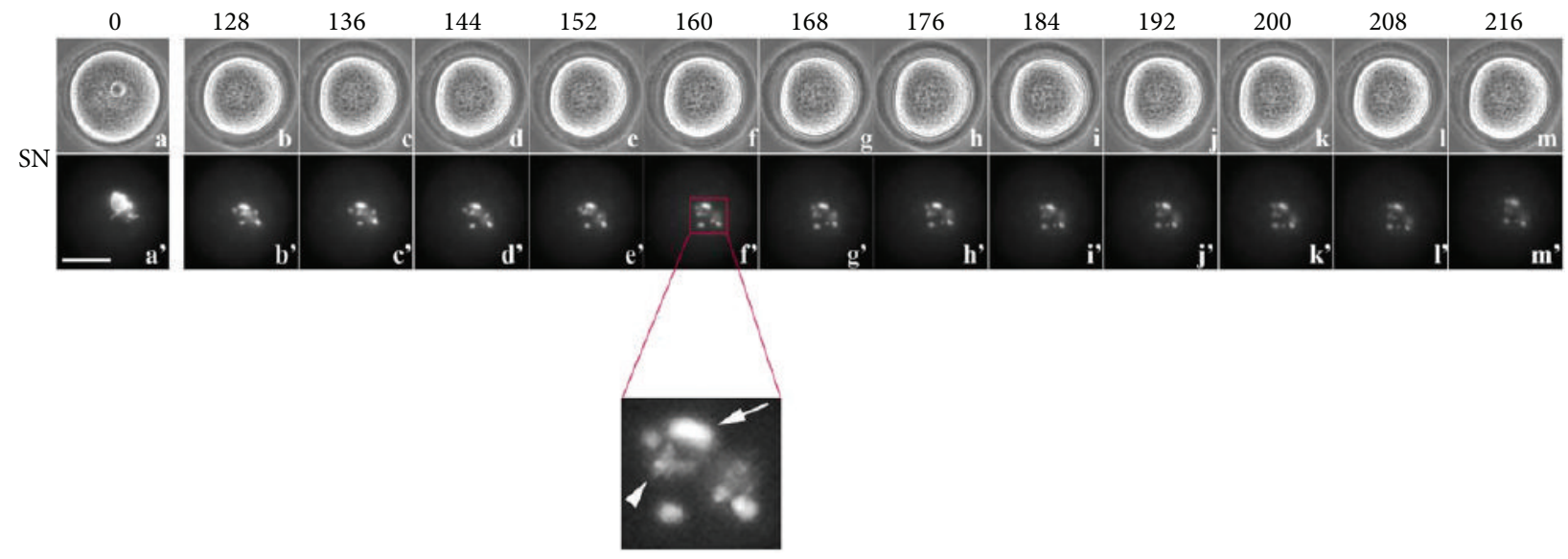

(a)

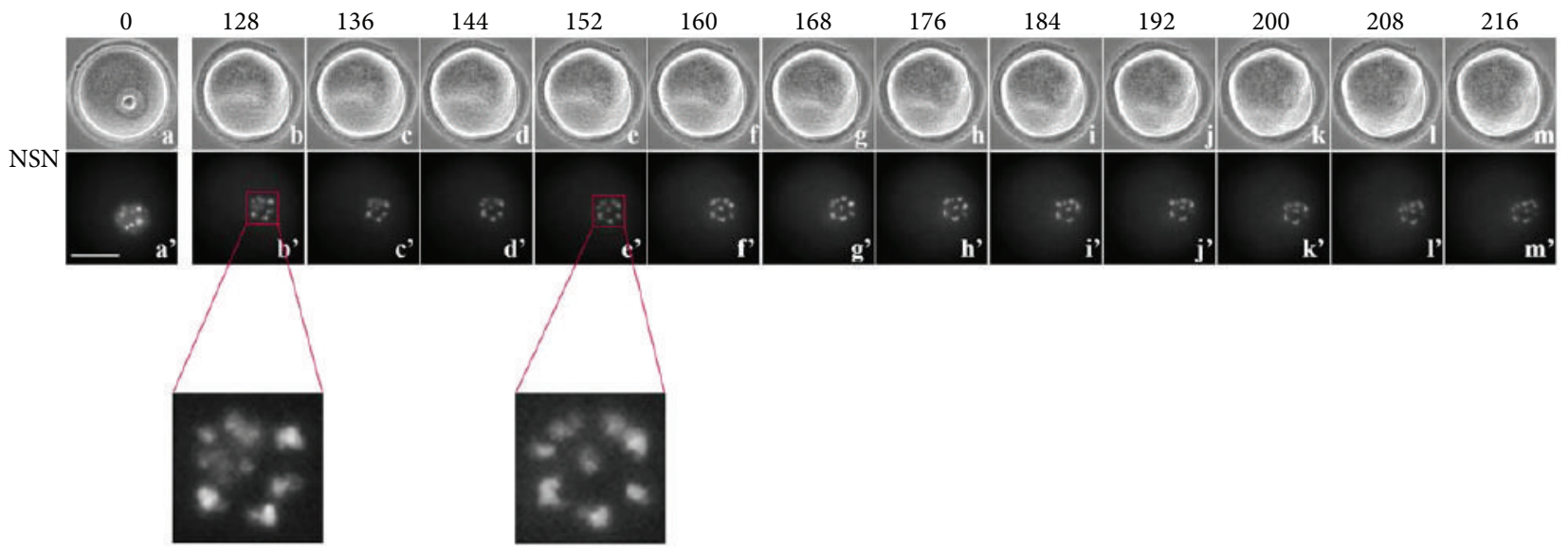

(b)

FIgURE 2: Time-lapse imaging from 128 to 216 minutes of oocyte culture. (a) SN oocyte, (a-m) bright field; (a'-m') UV fluorescence. Arrow in (f') enlargement, large CHC; arrowhead, small CHC. (b) NSN oocyte, (a-m) bright field; (a'-m') UV fluorescence. Bar: $40 \mu \mathrm{m}$.

The remaining $45.0 \%$ or $85.7 \%$ SN or NSN, respectively, attained the MII after the $9 \mathrm{hr}$ of time-lapse recording, within the 15 hr of IVM.

\section{Discussion}

In its reductionist layout, the experimental application of the SN/NSN model is a powerful tool that allows having, at one's disposal, ovarian oocytes of known developmental competence or incompetence. Staining of the chromatin with the fluorochrome Ho gives the opportunity to identify within a pool of oocytes isolated from the antral compartment of the ovary those that may develop to term (SN) from those that certainly arrest development soon after fertilisation (NSN) [25-28]. This early classification allowed us to focus on emerging differences between developmentally competent and incompetent oocytes, while they are maturing in vitro from the GV to the MII stage. A first difference that emerges is the significant longer time, almost doubled, that the NSN oocytes spend at the diplotene stage before undergoing GVBD (NSN: $\sim 51 \mathrm{~min}$ versus $\mathrm{SN}$ : $\sim 34 \mathrm{~min}$ ). When GVBD is almost completed and the nuclear envelope is dismantled, we observe a rim of black dots, seen under bright field in both types of oocytes, that represents mitochondria clearly visible for their autofluorescence when analysed at 440$490 \mathrm{~nm}$ wavelength ([34,35]; our unpublished observations). Then, by the time the oocyte reaches the MII phase, these mitochondria disperse within the ooplasm and become invisible when observed at the bright field ([34, 35]; our unpublished observations). These early events in meiosis resumption are accompanied by significant rearrangements of the cytoskeleton [36] that we could clearly record in SN, but less extensively in NSN oocytes, as a pulse contraction of the gamete itself. This shrinkage brings, as a consequence, to an enlargement of the perivitelline space on the one side of the oocyte, increase that is maintained as such throughout the remaining culture period.

The typical chromatin organisation of SN and NSN oocytes is maintained unaltered and distinct during the whole diplotene stage. Then, coincidentally with the GVBD and the beginning of diakinesis, the SN chromatin undergoes numerous changes, instead the NSN chromatin preserves its original organisation for much further (up $140 \mathrm{~min}$ ). The NSN nucleus maintains a steady chromatin organisation 


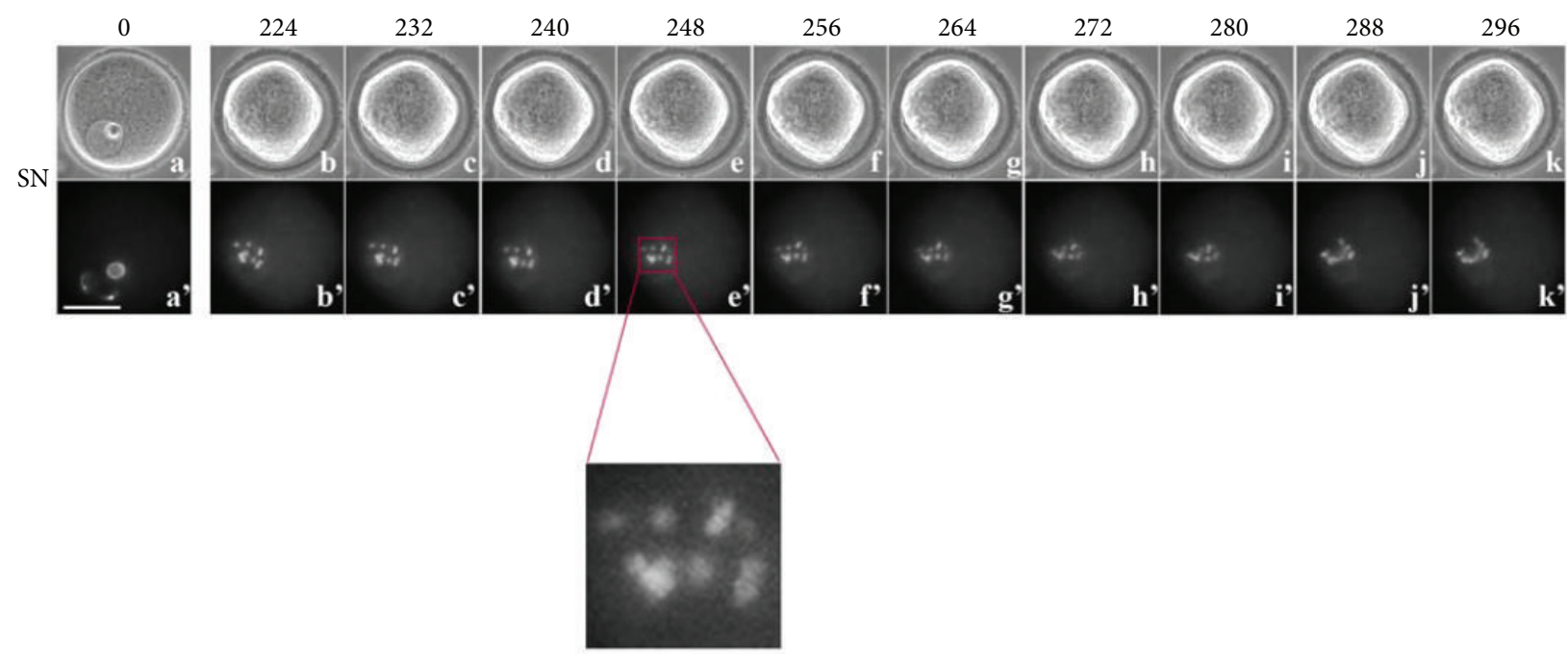

(a)
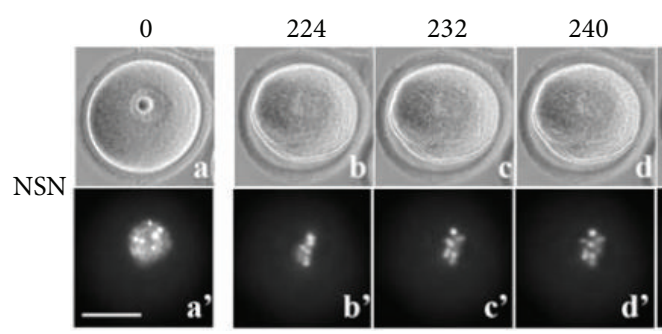

248

256

264

272

280

288

296

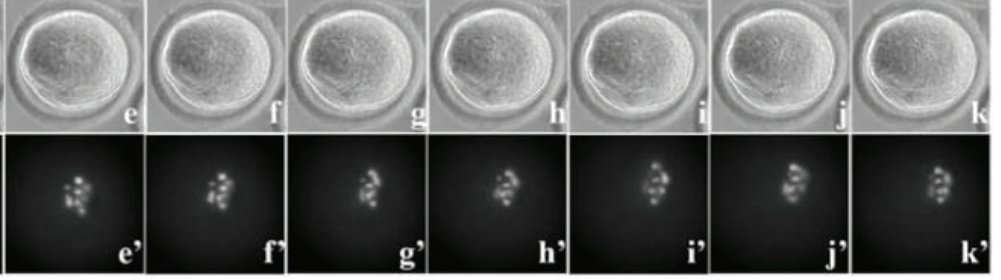

(b)

FIgURE 3: Time-lapse imaging from 224 to 296 minutes of oocyte culture. (a) SN oocyte, (a-k) bright field; (a'-k') UV fluorescence. (b) NSN oocyte, (a-k) bright field; (a'-k') UV fluorescence. Bar: $40 \mu \mathrm{m}$.

with 4/5 small Ho-positive CHCs around the nucleolus, corresponding to the pericentromeric area of NOR-bearing chromosomes [18]. Instead, the SN chromatin abruptly condenses ( 30 $\mathrm{min}$ ) around the nucleolar area; then, 4/5 CHCs emerge and become larger in size and more separated one from the other, marking the end of diakinesis and the beginning of the following prometaphase ( 100-120 min) [37]. The formation of the CHCs in SN oocytes has been explained with the gathering around the nucleolus of the pericentromeric regions of the 40 telocentric chromosomes of the mouse karyotype [18]. Then, these large CHCs become smaller in size and increase in number, likely as a consequence of the drifting away of chromosomes that later will begin to appear clearly visible as single entities arranged in a "rosette-like" organisation, a disposition of the chromosomes that marks the passage towards the MI phase.

Although these results show that the rosette-like figure is detected $\sim 80 \mathrm{~min}$ earlier in NSN compared to SN oocytes, the transition to MI occurs earlier in SN ( 350 min) compared to NSN ( 390 min) oocytes, suggesting a longer permanence in prometaphase for the latter gametes. The extended prometaphase in NSN oocytes may be explained with chromosome lagging in the congression towards the MI plate formation and may be causal to the about 4-fold higher aneuploidy rate that we described in NSN compared to SN oocytes in females that have undergone the same hormonal treatment [38]. The correlation between chromosome lagging and aneuploidy will be further analysed with a more detailed time-lapse analysis of the first meiotic division.

In summary, NSN oocytes undergo chromatin changes, distinct from those of SN oocytes, which prepare the genome to accomplish the following meiotic phases and reach MII. We observed a longer GV-to-MII transition in NSN oocytes that reach the $\mathrm{M}$-phase without the characteristic gathering of heterochromatin regions around the nucleolus [23]. Although we cannot identify a specific cause for the observed delay, a number of features that have been described may build up to a comprehensive picture of the biological nature of these two different antral oocytes. Interestingly, they display a different epigenetics status; that is, the SN chromatin configuration has higher levels of $\mathrm{CpG}$ methylation, histone acetylation (H4K5ac and $\mathrm{H} 4 \mathrm{~K} 12 \mathrm{ac})$, and methylation (H3K9me2) [39] which may be crucial to the dynamics of the large scale chromatin remodelling occurring soon after meiosis resumption. Interestingly, delayed transition was also described in SN oocytes treated with the histone deacetylase inhibitor trichostatin A [40], which prevents the onset of the global deacetylation occurring soon after meiosis resumption, indicating that perhaps NSN oocytes may present a lower or even damaged deacetylation activity. In addition to these described 


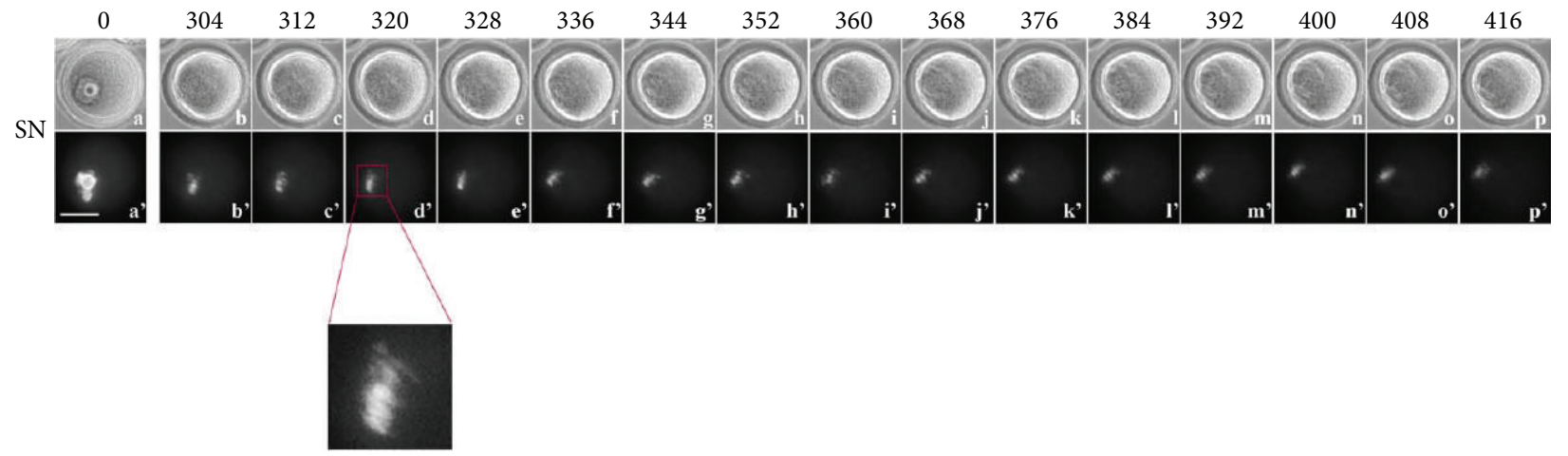

(a)

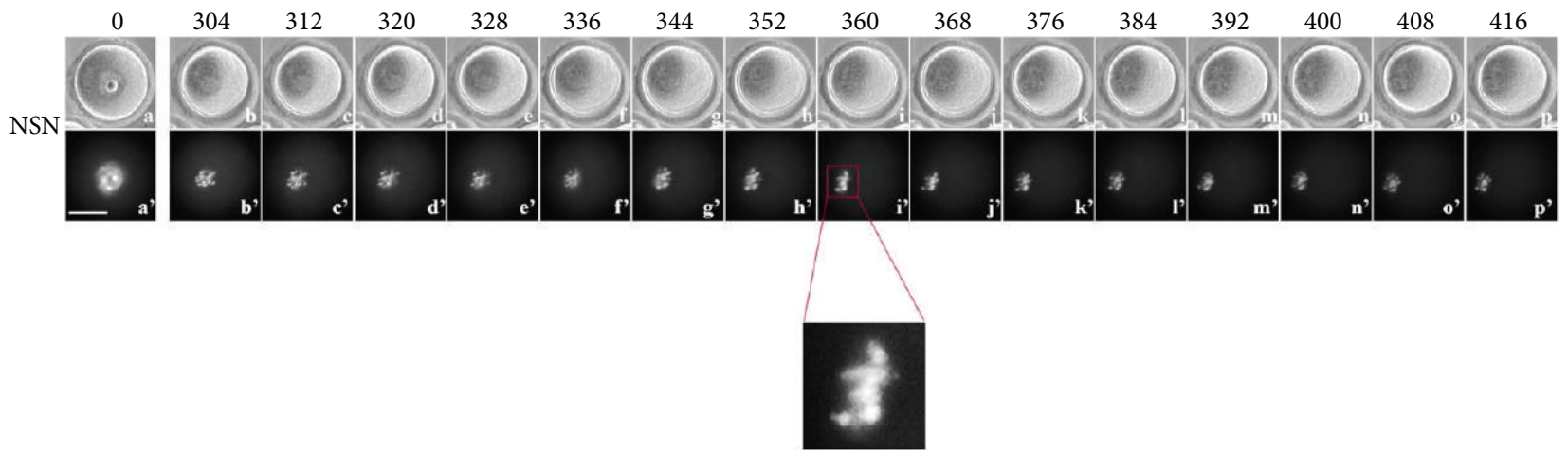

(b)

FIgURE 4: Time-lapse imaging from 304 to 416 minutes of oocyte culture. (a) SN oocyte, (a-p) bright field; (a'-p') UV fluorescence. (b) NSN oocyte, $(a-p)$ bright field; (a'-p') UV fluorescence. Bar: $40 \mu \mathrm{m}$.

differences, our own whole transcriptome microarrays studies show that NSN oocytes exhibit upregulation of transcriptional networks associated with mitochondrial dysfunction and apoptosis and downregulation of cell cycle transcripts [29].

When considered together with a number of recent molecular data, our morphological observations help to further understand the biological significance of these two types of oocytes within the ovary. Morphological and molecular data speak in favour of a separation of the maturation pathways of SN and NSN oocytes and possibly a distinct fate within the ovary. Microarrays studies demonstrated the presence in SN oocytes of a transcriptional network (TN) regulated by the oocyte-specific transcription factor OCT4 (OCT4-TN), whose downregulation in NSN oocytes plays a key function in a sequence of molecular events that lead to their developmental arrest [29]. From these studies, OCT4 emerges as a crucial regulator of the events that govern the establishment of the developmental competence of mouse oocytes [29, 31]. Mostly important is the pattern of expression of OCT4, which remains confined to oocytes with an SN type of chromatin configuration from the beginning to the end of oocyte growth, whereas it is downregulated in NSN oocytes throughout folliculogenesis [30]. A similar profile of expression is shown by other OCT4-regulated genes, including STELLA (DPPA3) [30], another oocyte-specific transcription factor whose lack of expression leads to a developmental arrest mainly at the 2-cell stage [30, 41].

\section{Conclusions}

In this study, we have minutely described and filmed, using time-lapse imaging, modifications to the oocyte morphology and to its chromatin organisation. The overall picture that comes to light is that of a pathway of transition from GV to MII for the two types of oocytes which is punctuated of discrete recordable events that show their specificity and occur with different time kinetics. The main significant differences recorded during oocyte maturation are (1) a reduction of the nuclear area that occurs before the GVBD, significant only for SN oocytes; (2) a 17 min delay of the GVBD in NSN oocytes; (3) an increased chromatin condensation, soon after the GVBD, that occurs only in SN oocytes; (4) the formation of 4-5 CHCs only in SN oocytes; (5) an increase of the perivitelline space that occurs $\sim 57$ min later in NSN oocytes; (6) the formation of a rosette-like disposition of the pericentromeric regions that takes place $\sim 84$ min later in SN oocytes; (7) the MI plate appears $\sim 40$ min later in NSN oocytes.

Altogether, morphological and molecular data of earlier studies build up to a model of mammalian ovary that 


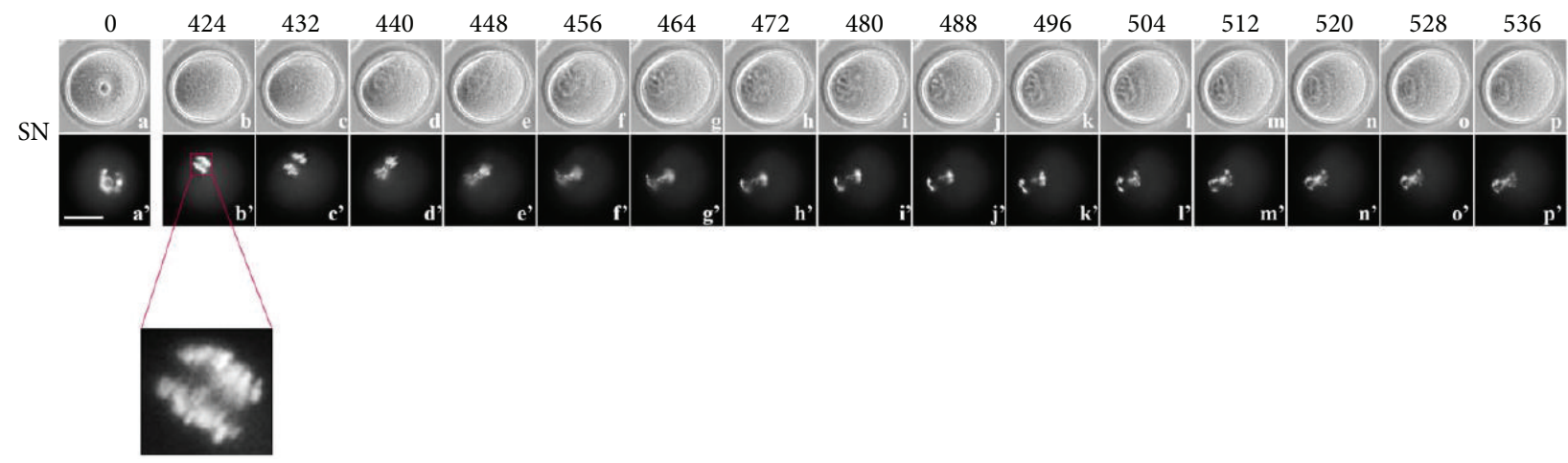

(a)

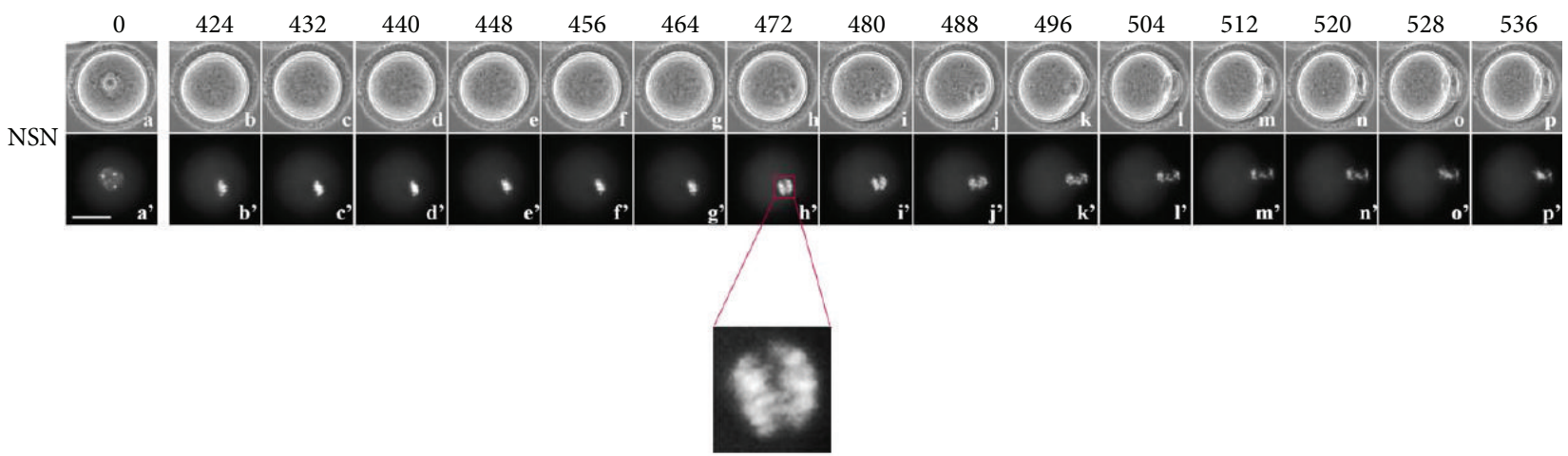

(b)

FIgURE 5: Time-lapse imaging from 424 to 536 minutes of oocyte culture. (a) SN oocyte, (a-p) bright field; (a'-p') UV fluorescence. (b) NSN oocyte, (a-p) bright field; (a'-p') UV fluorescence. Bar: $40 \mu \mathrm{m}$.

envisages the coexistence of follicles enclosing oocytes that are potentially developmentally competent or incompetent and both capable of growth and meiotic differentiation, at least in vitro. This model raises numerous questions, including whether growing follicles containing developmentally incompetent NSN oocytes may be rescued, that is, guided to acquire the SN chromatin organisation and whether this is paralleled by the acquisition of a developmental competent state. The answer to this question would improve our understanding of the yet poorly known biology of the mammalian ovary and would carry positive clinical implications to enhanced assisted reproductive technologies.

\section{Conflict of Interests}

The authors declare that there is no conflict of interests regarding the publication of this paper.

\section{Acknowledgments}

This study has been funded by the University of Pavia (FAR Grant) and the University of Parma (FIL Grant).

\section{References}

[1] T. Cremer, A. Kurz, R. Zirbel et al., "Role of chromosome territories in the functional compartmentalization of the cell nucleus," Cold Spring Harbor Symposia on Quantitative Biology, vol. 58, pp. 777-792, 1993.

[2] A. R. Leitch, "Higher levels of organization in the interphase nucleus of cycling and differentiated cells," Microbiology and Molecular Biology Reviews, vol. 64, no. 1, pp. 138-152, 2000.

[3] T. Cremer and C. Cremer, "Chromosome territories, nuclear architecture and gene regulation in mammalian cells," Nature Reviews Genetics, vol. 2, no. 4, pp. 292-301, 2001.

[4] L. A. Parada and T. Misteli, "Chromosome positioning in the interphase nucleus," Trends in Cell Biology, vol. 12, no. 9, pp. 425-432, 2002.

[5] S. M. Gasser, "Positions of potential: nuclear organization and gene expression," Cell, vol. 104, no. 5, pp. 639-642, 2001.

[6] T. Misteli, "Spatial positioning: a new dimension in genome function," Cell, vol. 119, no. 2, pp. 153-156, 2004.

[7] A. Taddei, F. Hediger, F. R. Neumann, and S. M. Gasser, "The function of nuclear architecture: a genetic approach," Annual Review of Genetics, vol. 38, pp. 305-345, 2004.

[8] H. A. Foster and J. M. Bridger, "The genome and the nucleus: a marriage made by evolution. Genome organisation and nuclear architecture," Chromosoma, vol. 114, no. 4, pp. 212-229, 2005. 
[9] V. Merico, J. Barbieri, M. Zuccotti et al., "Epigenomic differentiation in mouse preimplantation nuclei of biparental, parthenote and cloned embryos," Chromosome Research, vol. 15, no. 3, pp. 341-360, 2007.

[10] I. Alcobia, R. Dilao, and L. Parreira, "Spatial associations of centromeres in the nuclei of hematopoietic cells: evidence for cell-type-specific organizational patterns," Blood, vol. 95, no. 5, pp. 1608-1615, 2000.

[11] G. Martou and U. de Boni, "Nuclear topology of murine, cerebellar Purkinje neurons: changes as a function of development," Experimental Cell Research, vol. 256, no. 1, pp. 131-139, 2000.

[12] M. Cremer, K. Küpper, B. Wagler et al., "Inheritance of gene density-related higher order chromatin arrangements in normal and tumor cell nuclei," Journal of Cell Biology, vol. 162, no. 5, pp. 809-820, 2003.

[13] I. Solovei, L. Schermelleh, K. Düring et al., "Differences in centromere positioning of cycling and postmitotic human cell types," Chromosoma, vol. 112, no. 8, pp. 410-423, 2004.

[14] C. Martin, N. Beaujean, V. Brochard, C. Audouard, D. Zink, and P. Debey, "Genome restructuring in mouse embryos during reprogramming and early development," Developmental Biology, vol. 292, no. 2, pp. 317-332, 2006.

[15] M. Beil, D. Dürschmied, S. Paschke et al., "Spatial distribution patterns of interphase centromeres during retinoic acid-induced differentiation of promyelocytic leukemia cells," Cytometry, vol. 47, no. 4, pp. 217-225, 2002.

[16] I. Alcobia, A. S. Quina, H. Neves, N. Clode, and L. Parreira, "The spatial organization of centromeric heterochromatin during normal human lymphopoiesis: evidence for ontogenically determined spatial patterns," Experimental Cell Research, vol. 290, no. 2, pp. 358-369, 2003.

[17] I. A. Zalenskaya and A. O. Zalensky, "Non-random positioning of chromosomes in human sperm nuclei," Chromosome Research, vol. 12, no. 2, pp. 163-173, 2004.

[18] F. Longo, S. Garagna, V. Merico et al., "Nuclear localization of NORs and centromeres in mouse oocytes during folliculogenesis," Molecular Reproduction and Development, vol. 66, no. 3, pp. 279-290, 2003.

[19] S. Garagna, V. Merico, V. Sebastiano et al., "Three-dimensional localization and dynamics of centromeres in mouse oocytes during folliculogenesis," Journal of Molecular Histology, vol. 35, no. 6, pp. 631-638, 2004.

[20] C. Guetg and R. Santoro, "Formation of nuclear heterochromatin: the nucleolar point of view," Epigenetics, vol. 7, no. 8, pp. 811-814, 2012.

[21] T. Cremer and M. Cremer, "Chromosome territories," Cold Spring Harbor perspectives in biology, vol. 2, no. 3, Article ID a003889, 2010.

[22] D. Wickramasinghe, K. M. Ebert, and D. F. Albertini, "Meiotic competence acquisition is associated with the appearance of Mphase characteristics in growing mouse oocytes," Developmental Biology, vol. 143, no. 1, pp. 162-172, 1991.

[23] P. Debey, M. S. Szollosi, D. Szollosi, D. Vautier, A. Girousse, and D. Besombes, "Competent mouse oocytes isolated from antral follicles exhibit different chromatin organization and follow different maturation dynamics," Molecular Reproduction and Development, vol. 36, no. 1, pp. 59-74, 1993.

[24] M. Zuccotti, A. Piccinelli, P. G. Rossi, S. Garagna, and C. A. Redi, "Chromatin organization during mouse oocyte growth," Molecular Reproduction and Development, vol. 41, no. 4, pp. 479-485, 1995.
[25] M. Zuccotti, P. Giorgi Rossi, A. Martinez, S. Garagna, A. Forabosco, and C. A. Redi, "Meiotic and developmental competence of mouse antral oocytes," Biology of Reproduction, vol. 58, no. 3, pp. 700-704, 1998.

[26] M. Zuccotti, R. H. Ponce, M. Boiani et al., "The analysis of chromatin organisation allows selection of mouse antral oocytes competent for development to blastocyst," Zygote, vol. 10, no. 1, pp. 73-78, 2002.

[27] A. Inoue, R. Nakajima, M. Nagata, and F. Aoki, "Contribution of the oocyte nucleus and cytoplasm to the determination of meiotic and developmental competence in mice," Human Reproduction, vol. 23, no. 6, pp. 1377-1384, 2008.

[28] M. Bellone, M. Zuccotti, C. A. Redi, and S. Garagna, "The position of the germinal vesicle and the chromatin organization together provide a marker of the developmental competence of mouse antral oocytes," Reproduction, vol. 138, no. 4, pp. 639643,2009 .

[29] M. Zuccotti, V. Merico, L. Sacchi et al., "Maternal Oct-4 is a potential key regulator of the developmental competence of mouse oocytes," BMC Developmental Biology, vol. 8, article 97, 2008.

[30] M. Zuccotti, V. Merico, L. Sacchi et al., "Oct-4 regulates the expression of Stella and Foxj2 at the Nanog locus: implications for the developmental competence of mouse oocytes," Human Reproduction, vol. 24, no. 9, pp. 2225-2237, 2009.

[31] M. Zuccotti, V. Merico, M. Bellone et al., "Gatekeeper of pluripotency: a common Oct4 transcriptional network operates in mouse eggs and embryonic stem cells," BMC Genomics, vol. 12, article 345, 13 pages, 2011.

[32] M. Zuccotti, V. Merico, M. Belli et al., "OCT4 and the acquisition of oocyte developmental competence during folliculogenesis," International Journal of Developmental Biology, vol. 56, no. 10-12, pp. 853-858, 2012.

[33] J. Tan, H. Wang, X. Sun, Y. Liu, H. Sui, and J. Zhang, "Chromatin configurations in the germinal vesicle of mammalian oocytes," Molecular Human Reproduction, vol. 15, no. 1, pp. 1-9, 2009.

[34] R. Dumollard, M. Duchen, and J. Carroll, "The role of mitochondrial function in the oocyte and embryo," Current Topics in Developmental Biology, vol. 77, pp. 21-49, 2007.

[35] R. Dumollard, Z. Ward, J. Carroll, and M. R. Duchen, "Regulation of redox metabolism in the mouse oocyte and embryo," Development, vol. 134, no. 3, pp. 455-465, 2007.

[36] R. Li and D. F. Albertini, "The road to maturation: somatic cell interaction and self-organization of the mammalian oocyte," Nature Reviews Molecular Cell Biology, vol. 14, no. 3, pp. 141-152, 2013.

[37] S. M. Messinger and D. F. Albertini, "Centrosome and microtubule dynamics during meiotic progression in the mouse oocyte," Journal of Cell Science, vol. 100, no. 2, pp. 289-298, 1991.

[38] M. Zuccotti, M. Boiani, S. Garagna, and C. A. Redi, "Analysis of aneuploidy rate in antral and ovulated mouse oocytes during female aging," Molecular Reproduction and Development, vol. 50, no. 3, pp. 305-312, 1998.

[39] S. Kageyama, H. Liu, N. Kaneko, M. Ooga, M. Nagata, and F. Aoki, "Alterations in epigenetic modifications during oocyte growth in mice," Reproduction, vol. 133, no. 1, pp. 85-94, 2007. 
[40] F. Yang, C. Baumann, M. M. Viveiros, and R. de la Fuente, "Histone hyperacetylation during meiosis interferes with largescale chromatin remodeling, axial chromatid condensation and sister chromatid separation in the mammalian oocyte," International Journal of Developmental Biology, vol. 56, no. 1012, pp. 889-899, 2012.

[41] B. Payer, M. Saitou, S. C. Barton et al., "Stella is a maternal effect gene required for normal early development in mice," Current Biology, vol. 13, no. 23, pp. 2110-2117, 2003. 


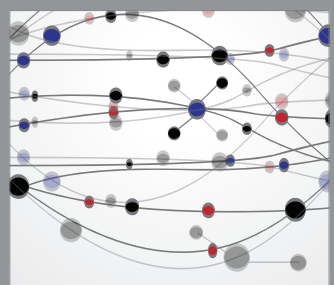

The Scientific World Journal
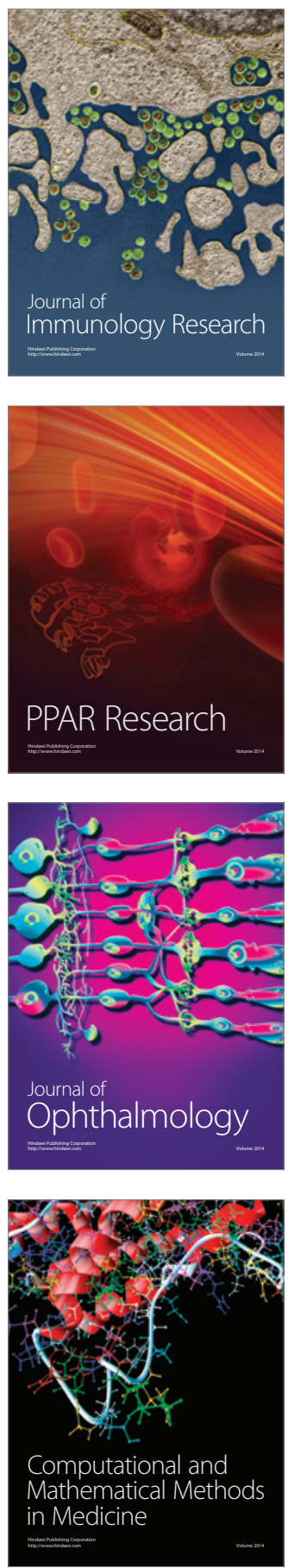

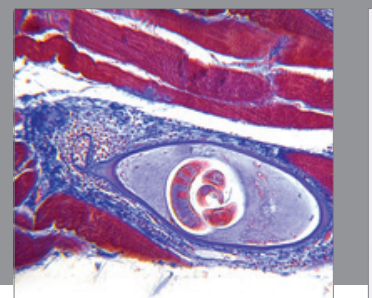

Gastroenterology

Research and Practice
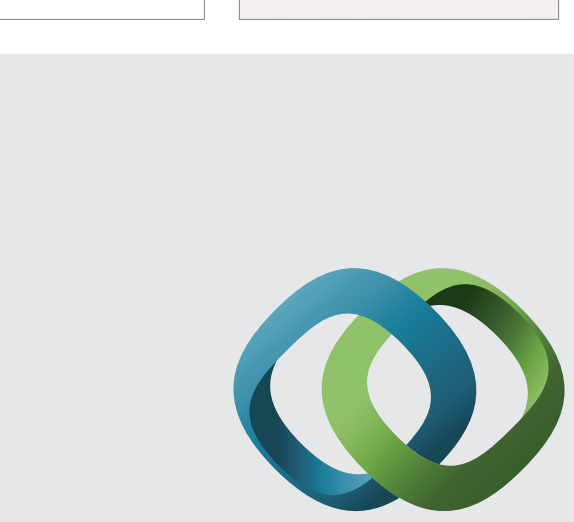

\section{Hindawi}

Submit your manuscripts at

http://www.hindawi.com
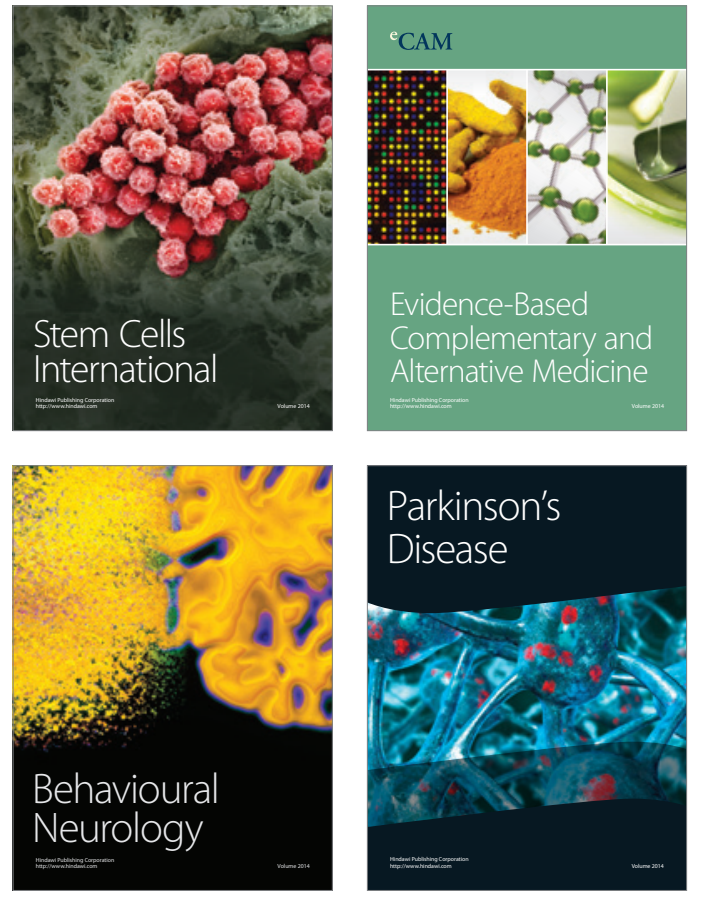
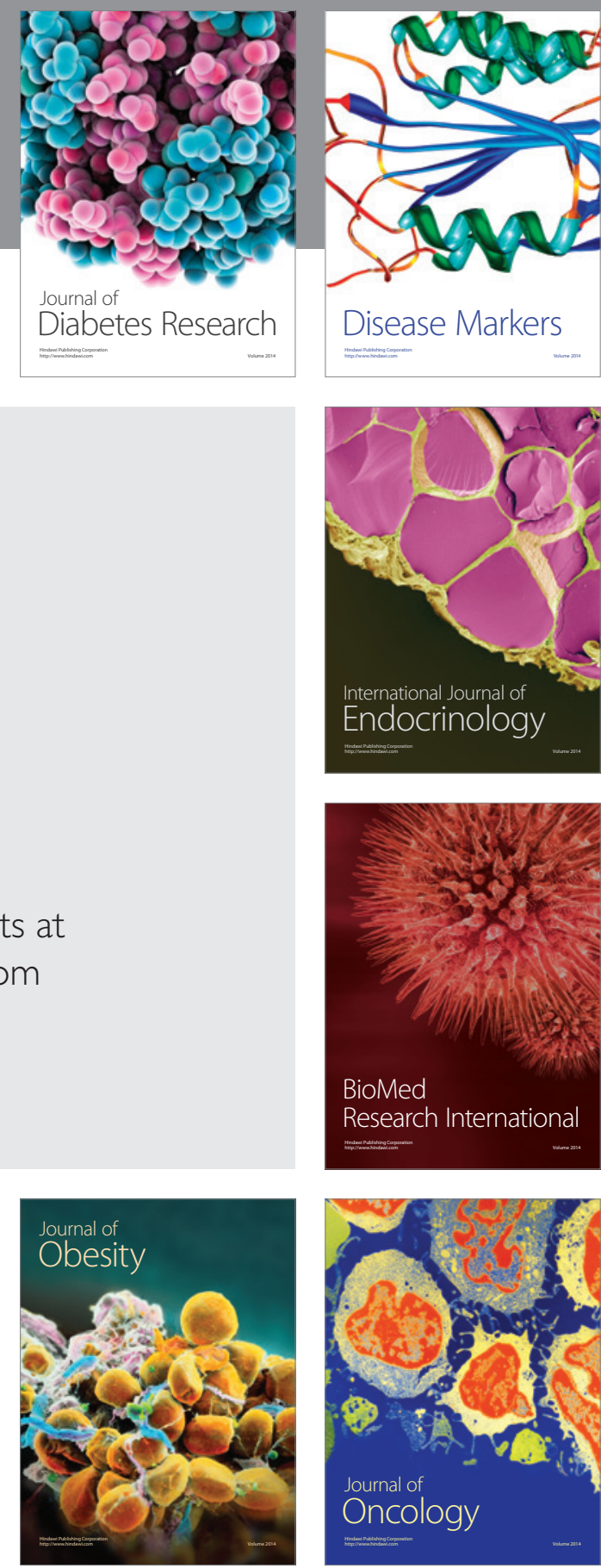

Disease Markers
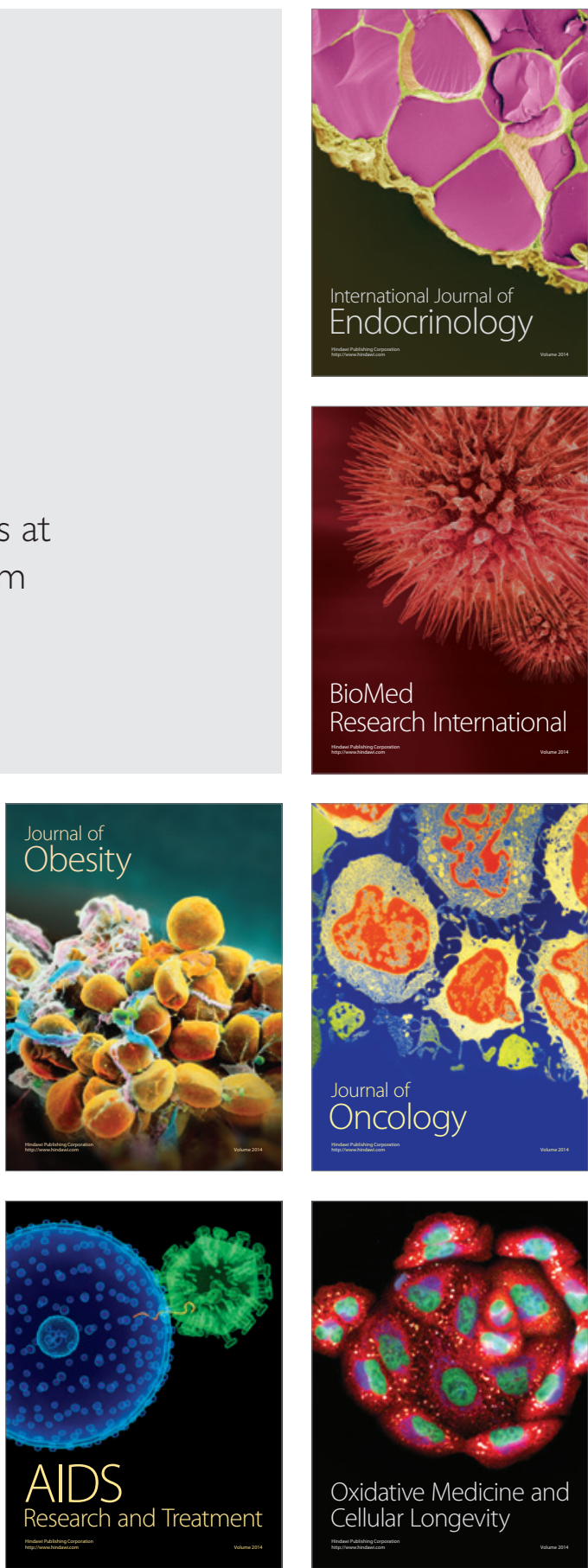